\title{
The Atlas Liquid Argon Calorimeter: Commissioning with Cosmic Muons and First LHC Beams
}

\author{
B.Trocméa , on behalf of the ATLAS Collaboration \\ ${ }^{a}$ LPSC, Université Joseph Fourier Grenoble 1, CNRS/IN2P3, INPG, Grenoble, France
}

In 2009, the Large Hadron Collider at CERN will collide protons with a center of mass energy of $14 \mathrm{TeV}$. ATLAS is a general purpose experiment that will allow to explore the wide potential of discovery and achieve high precision measurements. The ATLAS liquid argon calorimeters are presented, with an emphasis on their in situ commissioning using cosmic muons and their response during the first LHC single beam runs on September 2008 .

\section{The ATLAS liquid argon calorimeters}

The ATLAS liquid argon (LAr) calorimeters consist of four distinct sandwich calorimeters located in three cryostats and sharing the liquid argon as active medium[1]. The passive material and the geometry are specific to each part.

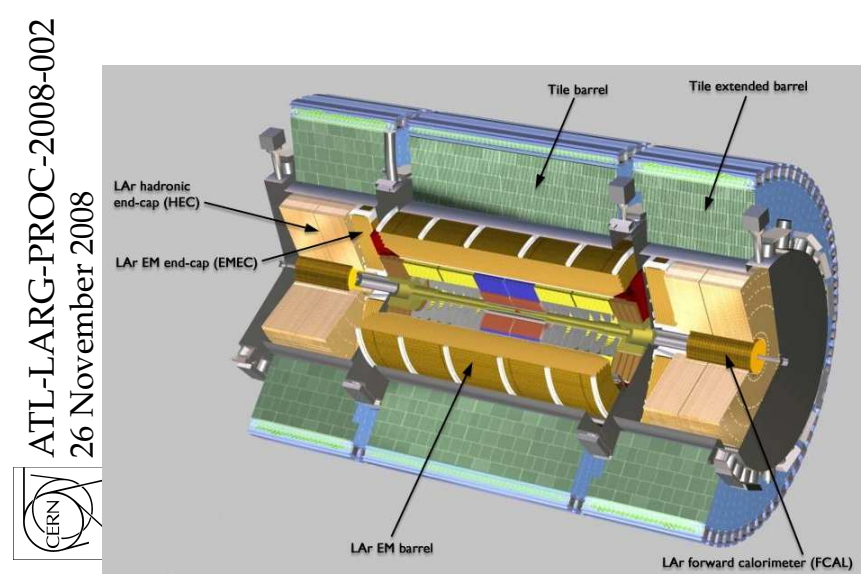

Figure 1. General view of the liquid argon calorimeters.

- the electromagnetic barrel and endcap calorimeters (EMB and EMEC) provide a precise measurement of electron and photon positions and energies up to a pseudo rapidity of 3.2. Their absorbers are made of lead, achieving a minimal radiation length of $22 X_{0}$. Their specific accordion geometry ensures a full hermiticity, a uniform and fast response. They are segmented in 3 distinct compartments in depth to extract the shower shape, with an additional presampler in order to estimate the loss due to the dead material in front of the calorimeter.

- the hadronic endcap (HEC) is a classical sandwich calorimeter with copper as passive material. Its pseudo rapidity coverage ranges from 1.5 to 3.2 , with a minimal interaction length of $10 \lambda$, segmented in depth in three compartments.

- the forward calorimeter (FCAL) detects the particles in the forward region with a pseudo rapidity coverage between 3.2 and 4.8. Due to the high particles occupancy in this region, a specific geometry with very thin liquid argon size gaps (between $250 \mu \mathrm{m}$ and $500 \mu \mathrm{m})$ has been adopted to limit the space charge, that could induce detection inefficiencies. The absorbers are made of tungsten (in first compartment) or copper (in second and third compartments), with a depth equivalent to $10 \lambda$.

The choice has been made to develop a unique readout system for the four different subdetectors. The signal produced by the electrodes is 
first routed to the front end boards (FEB) hosted in the crates installed on the cryostats. It is then treated for different purposes : after preamplification $^{1}$ and shaping, an analog sum is performed to be provided to the level 1 trigger system at a rate of $40 \mathrm{MHz}$. When a signal of level 1 trigger accept is received, the analog signal stored in a storage capacitance array (SCA) is digitized with a sampling rate up to $75 \mathrm{kHz}$. It is finally transferred through optical cables from the FEB to the readout driver cards (ROD) located in the counting rooms next to the detector cavern.

Depending on their configuration, the ROD cards either simply transfer the data to the central data acquisition system without further processing, or extract the energy, peaking time and quality factor by applying an optimal filtering method. The latter option is mandatory in LHC standard running due to bandwidth and storage space constraints; the first one is crucial during commissioning period to perform pulse shape studies and functionnality checks.

The individual gain factor for each cell can be extracted during a dedicated calibration procedure. A charge is injected very close to the location where the physical signal is produced in the electrode. Delay chips also allow to reconstruct the pulse shape with a time step of $1 \mathrm{~ns}$.

The performances of the LAr calorimeters have been extensively studied in several beam tests between 1999 and 2004. The last one consisted in installing a full slice of the ATLAS detector in the CERN SPS beam line[2]. During this period, a wide range of measurements confirmed that the LAr calorimeters were at the level of performances needed to fully exploit the LHC potential : the measured energy resolution of $\left.\sigma_{E} / E=(9.7 \pm 0.4) \% / \sqrt{(} E\right) \oplus(0.4 \pm 0.1) \%$ was found be in good agreement with the Monte Carlo prediction and well within specifications; the non linearity over a wide energy range (less than $0.4 \%$ over a a $250 \mathrm{GeV}$ range) and pseudo rapidity uniformity (less than $0.5 \%$ for $180 \mathrm{GeV}$ electrons) were also proven to be within specifications.

\footnotetext{
${ }_{1 \text { in the case of hadronic calorimeter, the preamplifiers are }}$ not located on the front end boards but directly in the cryostat
}

\section{Commissioning with muons}

Since summer 2006, cosmic muons data are regurlaly taken in order to check the functionning of the calorimeters, commission trigger and data acquisition systems, and to provide in situ performance measurements[3]. Muons, as minimum ionizing particles, deposit few hundred of $\mathrm{MeV}$ on average in the electromagnetic calorimeter. Depending on the trigger conditions (the trigger was derived from the tile calorimeter signal or from the muon detectors signal), the recorded event rate was observed to be around $0.1 \mathrm{~Hz}-1 \mathrm{~Hz}$, with a signal well above the noise level.

A selection on the minimal distance to ATLAS interaction point is applied in order to extract a sample of approximately 10000 pseudo projective muons and study detector uniformity. Figure 2 displays the reconstructed energy of clusters located in the pseudo-rapidity range $[0.3 ; 0.4]$. The

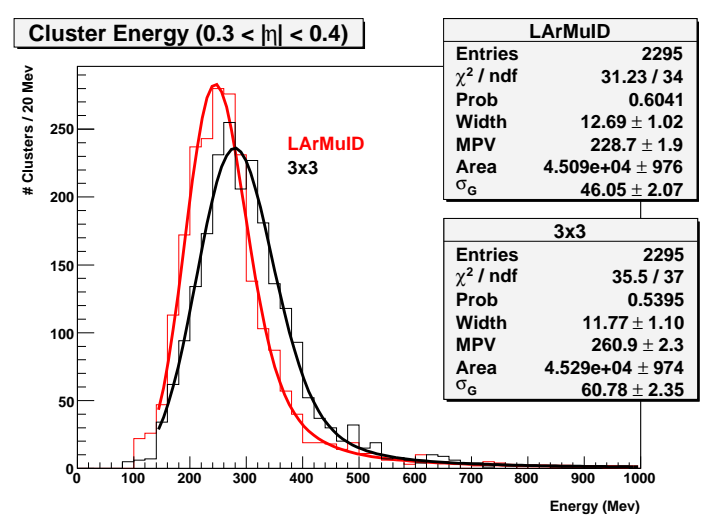

Figure 2. Distributions of energy of clusters for two clustering methods.

distributions, shown for two different clustering algorithms, can be fitted by a Landau distribution convoluted with a gaussian distribution. On figure 3 , are represented the variation of the fitted most probable value (MPV) of the Landau distribution as function of the pseudo rapidity for both the data and Monte Carlo simulation. If the 
Monte Carlo describes correctly the relative variation observed in the data, due to the variation of interaction lengths, an overall energy difference of $3 \%$ is observed. This difference is lower than the systematic uncertainty on the Monte Carlo energy normalization estimated around 5\%.

A very low fraction of muons also deposit a sig-

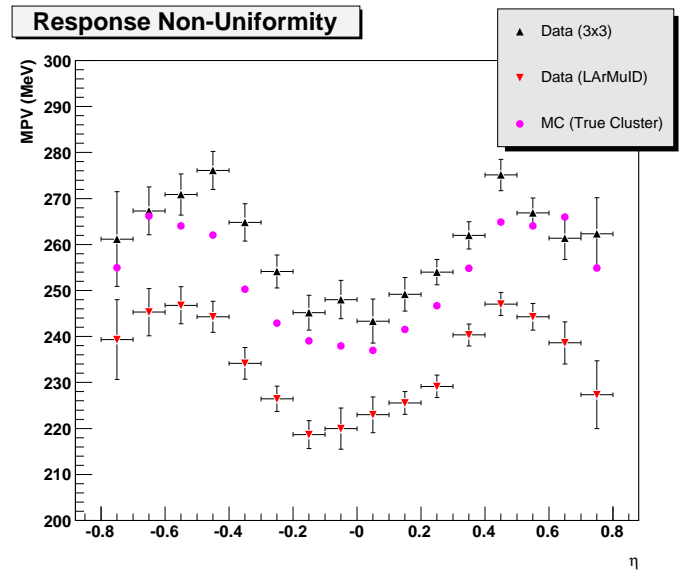

Figure 3. Pseudo rapidity variation of the fitted most probable value (MPV) parameter of the Landau fit for two clustering methods (data). The true cluster energy extracted from Monte Carlo is also displayed.

nificant amount of energy of few GeV[4]; in such large deposits, the contribution of gaussian noise becomes negligible : it then becomes possible to compare the measured pulse shape of the signal with its prediction, whose reliability is crucial to ensure an efficient optimal filtering method. An example for an electromagnetic cell is given on figure 4, exhibiting a very satisfactory agreement over the time range $0-600 \mathrm{~ns}$. The discrepancy in the range $600 \mathrm{~ns}-750 \mathrm{~ns}$ is explained by a small variation of the liquid argon gap size, but it does not affect the energy reconstruction, that only uses the peak region (here between 150ns and 250ns).

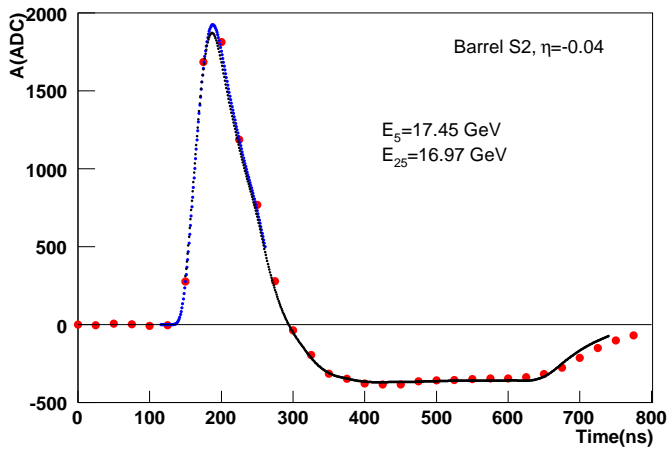

Figure 4. Typical cell response to high energy deposit in the second sampling of electromagnetic barrel. The blue (resp. black) curves correspond to the predicted pulse shape using 5 (resp. 25) time samples.

\section{Response to first LHC beams}

In September 2008, a $450 \mathrm{GeV}$ beam circulated in the Large Hadron Collider; despite the absence of collisions, the presence of collimators located in the LHC beam line at 140 meters from the ATLAS interaction point produced large showers of particles (pions, muons...) and induced sizable energy deposits in the LAr calorimeters. During this period, the LAr calorimeters were operated with an almost full efficiency ${ }^{2}$ : less than $1 \%$ of the total 170000 readout channels were indeed not operational (after winter 2009 maintenance operations, the number of dead readout channels is expected to be even lower than $0.02 \%$ ). Several sources of signal were used to trigger the data acquisition : minimum bias scintillator installed on the endcap cryostats, calorimeter level 1 trigger system and beam position monitor located $175 \mathrm{~m}$ from the ATLAS interaction point. In the following, are considered the 86 events triggered by the beam position monitors during a run taken on 13th of September (Atlas run number : 87851).

Figure 5 displays the accumulated energy deposited in the second sampling of the electro-

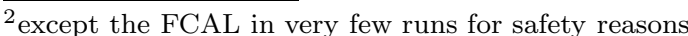




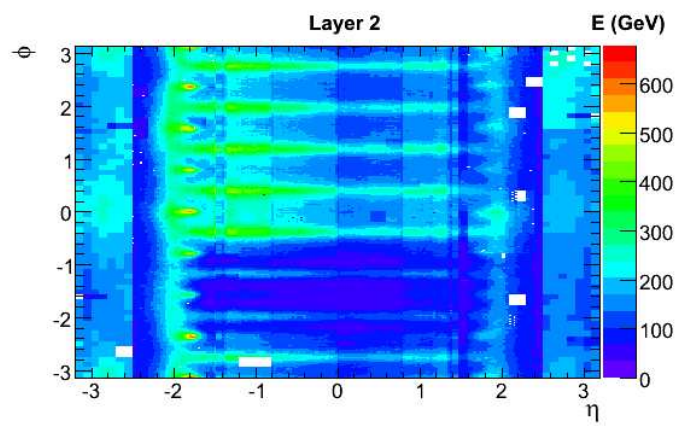

Figure 5. $(\eta, \phi)$ map of energy deposited in second sampling of electromagnetic calorimeters (accumulated over 86 events).

magnetic calorimeter as a function of the pseudorapidity and azymutal coordinate. The observed $\phi$ modulation, with eight energy dips, is due to the presence of the toroid endcap magnet located between the collimators and the LAr detectors. The lower energy deposit in the $\phi$ region $[-2 ;-1]$ (corresponding to the lower part of the detector) can be explained by the LHC tunnel geometry, with an enhanced screening of particle flow in this lower region. With a very preliminary energy calibration and uncertain beam conditions, the absolute energy scale is not really relevant.

The pseudo-rapidity variation of the energy deposit in the different samplings of the electromagnetic calorimeters is displayed on Figure 6 . The structure with several gaps reflects the interaction length variation with pseudo-rapidity, whereas the asymetry between positive and negative coordinates is explained by the asymetrical nature of the (single) beam conditions.

\section{Conclusion}

The liquid argon calorimeters of ATLAS are installed in the experimental area since winter 2007. After 10 years of development, several beam tests and in situ commissioning with cosmic muons, they were fully operational for the first runs of

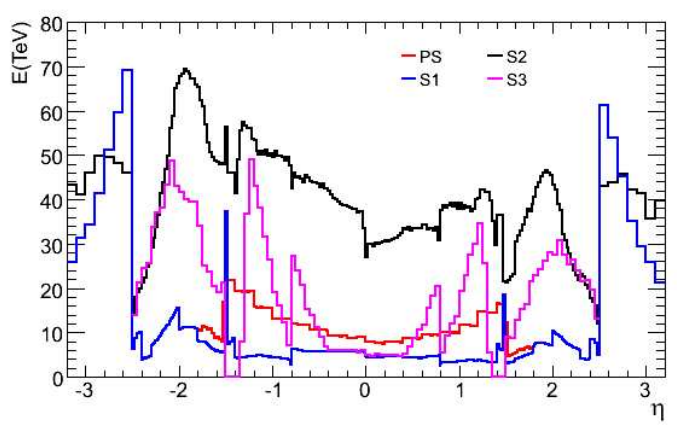

Figure 6. Pseudo rapidity variation of energy deposited in four samplings of electromagnetic calorimeters (accumulated over 86 events).

LHC commissioning in September 2008. The preliminary results obtained from single beam events are promising and further studies should allow a better understanding of their behaviours, in order to exploit fruitfully the first collisions in 2009.

\section{Acknowledgements}

The work presented here has been performed within the ATLAS LAr collaboration. It would not have been possible without the dedicated effort of many people in our LAr detector group and the ATLAS collaboration over many years. I am especially indebted to people who built, integrated and installed the LAr dectectors in the ATLAS cavern and those who operate the detector on a daily basis.

\section{REFERENCES}

1. The ATLAS Collaboration, G. Aad et al., JINST 3 (2008) S08003.

2. M. Aharrouche et al, ATLAS-LARG-PUB to be published (2009)

3. M. Cook et al, ATLAS-LARG-PUB-2007-013 (2007)

4. C. Gabaldon Ruiz et al, ATLAS-LARG-PUB2008-001 (2008) 